Canad. Math. Bull. Vol. 21 (1), 1978

\title{
UNIFORM CONTRACTIFICATION
}

\author{
BY \\ KOK-KEONG TAN ${ }^{(1)}$
}

\begin{abstract}
Let $(X, \tau)$ be a metrizable space and $\left\{f_{n}: n=1,2, \ldots\right\}$ be a commuting family of continuous mappings on $X$ with a common fixed point $\xi \in X$ such that (I) for each $k=$ $1,2, \ldots, f_{k}^{n}[X] \rightarrow\{\xi\}$ as $n \rightarrow \infty$ and (II) $\cup_{n \geq k} f_{n}[X] \rightarrow\{\xi\}$ as $k \rightarrow \infty$. Then for each $c \in(0,1)$, there exists a metric $d$ on $X$ inducing the topology $\tau$ such that $d\left(f_{n}(x), f_{n}(y)\right) \leq c d(x, y)$, for all $x, y \in X$ and $n=1,2, \ldots$. The above result is also generalized to Tychonoff spaces.
\end{abstract}

Let $(X, \tau)$ be a Tychonoff space and $h(\tau)$ be the collection of all families of pseudometrics on $X$ inducing $\tau$. If $A \subset X, \bar{A}$ denotes the closure of $A$. If $D \in h(\tau)$, a sequence $\left(x_{n}\right)_{n=1}^{\infty}$ in $X$ is said to be a Cauchy sequence with respect to $D$ if for each $d \in D, d\left(x_{n}, x_{m}\right) \rightarrow 0$ as $n, m \rightarrow \infty .(X, \tau)$ is sequentially complete with respect to $D$ if every Cauchy sequence with respect to $D$ converges in $(X, \tau)$.

THeOREM 1. Let $(X, \tau)$ be a Thchonoff space and $\left\{f_{n}: n=1,2, \ldots\right\}$ a commuting family of continuous mappings on $X$ with a common fixed point $\xi \in X$ such that

(i) for each $k=1,2, \ldots, f_{k}^{n}[X] \rightarrow\{\xi\}$ as $n \rightarrow \infty$

(ii) $\cup_{n \geq k} f_{n}[X] \rightarrow\{\xi\}$ as $k \rightarrow \infty$.

Then for each $D \in p(\tau)$, there exists $D^{*} \in p(\tau)$ such that (i) Card $D=\operatorname{Card} D^{*}$, and (ii) for each $\rho \in D^{*}, \rho \leq 1$ and $\rho\left(f_{n}(x), f_{n}(y)\right) \leq \rho(x, y)$, for all $x, y \in X$ and $n=1,2, \ldots$ Moreover, if $(X, \tau)$ is sequentially complete with respect to $D,(X, \tau)$ is also sequentially complete with respect to $D^{*}$.

Proof. Let $D \in p(\tau)$. We may assume that $d \leq 1$ for each $d \in D$, otherwise we replace $d$ by the equivalent pseudometric $d / 1+d$. For each $d \in D$, define

$$
d^{*}(x, y)=\sup \left\{d\left(f_{1}^{k_{1}} \cdots f_{n}^{k_{n}}(x), f_{1}^{k_{1}} \cdots f_{n}^{k_{n}}(y)\right): k_{i} \geq 0, n=1,2, \ldots\right\}
$$

for all $x, y \in X$. Let $D^{*}=\left\{d^{*}: d \in D\right\}$. Then $D^{*}$ satisfies all the required properties. (See the proof of Theorem 1 in [1].) The last assertion follows from the fact that $d \leq d^{*}$ for all $d \in D$. A-8096.

(1) The author is partially supported by the National Research Council of Canada under grant Received by the editors March 3, 1977 and revised May 10, 1977. 
Corollary 2. Let $(X, \tau)$ be a metrizable space and $\left\{f_{n}: n=1,2, \ldots\right\}$ be a commuting family of continuous mappings on $X$ with a common fixed point $\xi \in X$ such that the conditions ( $i)$ and (ii) in Theorem 1 are satisfied. Then there exists a bounded metric $d(\leq 1)$ on $X$ inducing $\tau$ such that $d\left(f_{n}(x), f_{n}(y)\right) \leq d(x, y)$, for all $x, y \in X$ and $n=1,2, \ldots$ Moreover, the metric $d$ can be chosen complete if $(X, \tau)$ is completely metrizable.

In the proof of the next theorem, we use the idea first developed in the proof of theorem 2 in [4].

THEOREM 3. Let $(X, \tau)$ be a metrizable space and $\left\{f_{n}: n=1,2, \ldots\right\}$ be a commuting family of continuous mappings on $X$ with a common fixed point $\xi \in X$ such that the conditions $(i)$ and (ii) in Theorem 1 are satisified. Then $\left\{f_{n}: n=\right.$ $1,2, \ldots\}$ is uniformly contractifiable under a bounded metric on $X$, i.e., for each $c \in(0,1)$, there exists a bounded metric $d(\leq 1)$ on $X$ inducing $\tau$ such that $d\left(f_{n}(x), f_{n}(y)\right) \leq c d(x, y)$, for all $x, y \in X$ and $n=1,2, \ldots$.

Proof. By Corollary 2 , there exists a bounded metric $\rho(\leq 1)$ on $X$ inducing $\tau$ such that $\rho\left(f_{n}(x), f_{n}(y)\right) \leq \rho(x, y)$, for all $x, y \in X$ and $n=1,2, \ldots$ For $x, y \in X$, let

$$
\begin{aligned}
n(x) & =\sup \left\{m: m=n_{1}+\cdots+n_{k}, \text { where } n_{i} \geq 0, k \geq 1 \text { and } x \in \overline{f_{1}^{n_{1}} \cdots f_{k}^{n_{k}}[X]}\right\}, \\
n(x, y) & =\min \{n(x), n(y)\}, \\
\lambda(x, y) & =c^{n(x, y)} \rho(x, y), \text { with the convention that } c^{\infty}=0, \\
d(x, y) & =\inf \left\{\sum_{i=1}^{n} \lambda\left(x_{i}, x_{i+1}\right): x_{1}, \ldots, x_{n+1} \in X \text { with } x_{1}=x\right. \text { and } \\
x_{n+1} & =y, n=1,2, \ldots\} .
\end{aligned}
$$

Then we can show that $(a) d$ is a bounded (by 1) metric on $X$ such that $d\left(f_{n}(x), f_{n}(y)\right) \leq c d(x, y)$, for all $x, y \in X$ and $n=1,2, \ldots$ and $(b)$ for all $x, y \in X \quad$ with $\quad x \neq \xi, \quad d(x, y) \geq c^{c(x)} \cdot \min \left\{L_{x}, \rho(x, y)\right\}, \quad$ where $L_{x}=$ $\inf \left\{\rho\left(x, \overline{\left.f_{1}^{t_{1}} \cdots f_{q}^{t_{q}}[X]\right)}: q \geq 1\right.\right.$ and $\left.t_{1}+\cdots+t_{q}>n(x)\right\}>0$. (See the proof of Theorem 3 in [1].) To complete the proof, it remains to prove that $d$ and $\rho$ are equivalent. Since $d \leq \rho$, it suffices to show that for $x_{n}, x \in X, n=$ $1,2, \ldots, d\left(x, x_{n}\right) \rightarrow 0$ as $n \rightarrow \infty$ implies $\rho\left(x, x_{n}\right) \rightarrow \infty$ as $n \rightarrow \infty$.

CASE 1. Suppose $x \neq \xi$. Then by $(b), d\left(x, x_{n}\right) \geq c^{n(x)} \cdot \min \left\{L_{x}, \rho\left(x, x_{n}\right)\right\}$, where $L_{x}>0$ depends only on $x$. Since $d\left(x, x_{n}\right) \rightarrow 0$ as $n \rightarrow \infty$, we must have $\rho\left(x, x_{n}\right) \rightarrow \infty$.

Before we prove the other case, we shall prove the following:

$\left(^{*}\right)$ for any $\varepsilon>o$, there exists a positive integer $N(\varepsilon)$ such that for all $y \in X$, $\rho(y, \xi)>\varepsilon$ implies $d(y, \xi) \geq c^{N(\varepsilon)} \cdot \varepsilon / 2$. Indeed, let $\varepsilon>0$. Since $\cup_{n \geq k} f_{n}[X] \rightarrow\{\xi\}$ as $k \rightarrow \infty$, there exists a positive integer $N_{0}>1$ such that $\overline{U_{n \geqslant N_{0}} f_{n}[X]} \subset B_{\rho}(\xi ; \varepsilon / 2)=\{z \in X: \rho(\xi, z)<\varepsilon / 2\}$. For each $k=1, \ldots, N_{0}-1$, 
since $f_{k}^{n}[X] \rightarrow\{\xi\}$ as $n \rightarrow \infty$, there exists a positive integer $n_{k}$ such that $\overline{f_{k}^{n_{k}}[X]} \subset$ $B_{\rho}(\xi ; \varepsilon / 2)$. Define $N(\varepsilon)=n_{1}+\cdots+n_{N_{0}-1}$. Note that for any $z \in X$, $z \notin \overline{\cup_{j \geq N_{0}} f_{j}[X] \cup \cup_{i=1}^{N_{0}-1} f_{i}^{n_{i}}[X]}$ implies $n(z)<N(\varepsilon)$. Now suppose $\rho(y, \xi)>\varepsilon$. Let $\eta>0$ be given. Then there exists $x_{1}=y, x_{2}, \ldots, x_{M+1}=\xi \in X$ such that $d(y, \xi)+\eta>\sum_{i=1}^{M} c^{n^{\prime}\left(x_{i}, x_{i+1}\right)} \rho\left(x_{i}, x_{i+1}\right)$. Define $k=\min \left\{i: \rho\left(x_{i}, \xi\right)<\varepsilon / 2\right\}$, then $k \geq 2$ since $\rho(y, \xi)>\varepsilon$. It follows that for $i=1, \ldots, k-1, \rho\left(x_{i}, \xi\right) \geq \varepsilon / 2$ and hence $n\left(x_{i}\right)<N(\varepsilon)$. Thus

$$
\begin{aligned}
d(y, \xi)+\eta \geq & c^{N(\varepsilon)} \rho\left(y, x_{2}\right)+\cdots+c^{N(\varepsilon)} \rho\left(x_{k-1}, x_{k}\right)+c^{n\left(x_{k}, x_{k+1}\right)} \rho\left(x_{k}, x_{k+1}\right) \\
& +\cdots+c^{n\left(x_{M}, \xi\right)} \rho\left(x_{M}, \xi\right) \\
\geq & c^{N(\varepsilon)} \cdot\left\{\rho\left(y, x_{2}\right)+\cdots+\rho\left(x_{k-1}, x_{k}\right)\right\} \\
\geq & c^{N(\varepsilon)} \cdot \rho\left(y, x_{k}\right) \\
\geq & c^{N(\varepsilon)} \cdot \frac{\varepsilon}{2}
\end{aligned}
$$

Therefore $d(y, \xi) \geq c^{N(\varepsilon)} \cdot \varepsilon / 2$ as $\eta>0$ is arbitrary.

CASE 2. Suppose $x=\xi$, i.e. $d\left(x_{n}, \xi\right) \rightarrow 0$ as $n \rightarrow \infty$. Thus for $\varepsilon>0$, there exists a positive integer $N$ such that $d\left(x_{n}, \xi\right)<c^{N(\varepsilon)} \cdot \varepsilon / 2$ for all $n \geq N$. By $\left(^{*}\right)$, $\rho\left(x_{n}, \xi\right) \leq \varepsilon$ for all $n \geq N$. Hence $\rho\left(x_{n}, \xi\right) \rightarrow 0$ as $n \rightarrow \infty$. This completes the proof of the theorem.

The above result solves the problem posted at the end of section 1 in [2].

EXAmple 4. Let $X=[0,1]$ equipped with the usual topology $\tau$. For $n=$ $1,2, \ldots$, define $f_{n}(x)=\left(1-\frac{1}{n}\right) x$, for all $x \in X$. Then $\left\{f_{n}: n=1,2, \ldots\right\}$ is a commuting family of continuous mappings on $X$ with a common fixed point $\xi=0$ and satisfies the condition (i) but not the condition (ii) in Theorem 3 . Since $f_{k}^{n}[X] \rightarrow\{0\}$ as $n \rightarrow \infty$ is not uniform in $k,\left\{f_{n}: n=1,2, \ldots\right\}$ cannot be uniformly contractifiable, i.e. there does not exist a metric $d$ on $X$ equivalent to the usual metric on $X$ such that each $f_{n}$ is a $d$-contraction with the same Lipschitz constant.

If $X$ is the real line, the above example was given by A. J. Goldman and P. $R$. Meyers in [2]. Since [0,1] is compact, the above examples shows that even if $(X, \tau)$ is compact, the condition (ii) in Theorem 3 (and hence also Theorem 3 in [1]) cannot be omitted.

Observing that if $\left\{f_{n}: n=1,2, \ldots\right\}$ is uniformly contractifiable under a bounded metric, then $f_{k}^{n}[X] \rightarrow\{\xi\}$ as $n \rightarrow \infty$ uniformly in $k$, Theorem 3 can be rephrased to give us the following characterizations:

Theorem 5. Let $(X, \tau)$ be a metrizable space and $\left\{f_{n}: n=1,2, \ldots\right\}$ be a commuting family of continuous mappings on $X$ with a common fixed point $\xi \in X$ such that $\cup_{n \geq k} f_{n}[X] \rightarrow \infty$. Then the following are equivalent:

(1) For each $k=1,2, \ldots, f_{k}^{n}[X] \rightarrow\{\xi\}$ as $n \rightarrow \infty$. 
(2) $f_{k}^{n}[X] \rightarrow\{\xi\}$ as $n \rightarrow \infty$, uniformly in $k$,

(3) $\left\{f_{n}: n=1,2, \ldots\right\}$ is uniformly contractifiable under a bounded metric on $X$.

Theorems 1 and 3 together with necessary modification in the proof of Theorem 4 in [1] give us the following:

Theorem 6. Let $(X, \tau)$ be a Tychonoff space and $\left\{f_{n}: n=1,2, \ldots\right\}$ be a commuting family of continuous mappings on $X$ with a common fixed point $\xi \in X$ such that $\cup_{n \geq k} f_{n}[X] \rightarrow\{\xi\}$ as $k \rightarrow \infty$. Then the following are equivalent:

(1) for each $k=1,2, \ldots, f_{k}^{n}[X] \rightarrow\{\xi\}$ as $k \rightarrow \infty$,

(2) $f_{k}^{n}[X] \rightarrow\{\xi\}$ as $n \rightarrow \infty$, uniformly in $k$,

(3) $\left\{f_{n}: n=1,2, \ldots\right\}$ is topologically uniformly contractifiable under bounded pseudometrics on $X$, i.e. for each $c \in(0,1)$ and for each $D \in p(\tau)$, there exists $D^{*} \in p(\tau)$ such that $(i)$ Card $D=$ Card $D^{*}$, and (ii) for each $\rho \in D^{*}, \rho \leq 1$, and $\rho\left(f_{n}(x), f_{n}(y)\right) \leq c \rho(x, y)$, for all $x, y \in X$ and $n=1,2, \ldots$.

If $f$ is a mapping on $\mathrm{X}$, define $f_{k}=f^{k}$ for $k=1,2, \ldots$, we see that the conditions (i) and (ii) coincide. This observation gives us the following:

Corollary 7. Let $(X, \tau)$ be a Tychonoff space and $f: X \rightarrow X$ be continuous with a fixed point $\xi \in X$. Then the following are equivalent:

(1) $f^{n}[X] \rightarrow\{\xi\}$ as $n \rightarrow \infty$,

(2) for each $c \in(0,1)$ and for $D \in p(\tau)$ there exists $D^{*} \in p(\tau)$ such that $(i)$ Card $D=$ Card $D^{*}$ and (ii) for each $\rho \in D^{*}, \rho \leq 1$ and $\rho(f(x), f(y)) \leq c \rho(x, y)$ for all $x, y \in X$.

The above result answers the question raised at the end of [3].

We conclude here with the following two open problems:

Problem 1. In Theorem 3 , if $(X, \tau)$ is completely metrizable, can the metric $d$ be so chosen to be also complete?

We remark that the above question remains open even if $\left\{f_{n}: n=1,2, \ldots\right\}$ is finite.

Problem 2. In Theorem 5 (resp. Theorem 6), if we drop the condition " $\cup_{n \geq k} f_{n}[X] \rightarrow\{\xi\}$ as $k \rightarrow \infty$," do conditions (2) and (3) remain equivalent?

\section{REFERENCES}

1. K. P. Chew and K. K. Tan, Remetrization and family of commuting contractive type mappings, to appear in the Proceedings of the Seminar on fixed point theory and applications, Dalhousie University, 1975.

2. A. J. Goldman and P. R. Meyers, Simultaneous contractification, J. Res. NBS, 73B (Math. and Math. Phys.) No. 4, 301-305. 
3. L. Janos, Contraction property of the operator of integration, Can. Math. Bull. 18 (1975), 367-369.

4. L. Jones, A converse of Banach's contraction theorem, Proc. Amer. Math. Soc. 18 (1967), 287-289.

\section{Mathematics Department \\ DALHOUSIE UNIVERSITY \\ Halifax, Nova Scotia \\ CANADA B3H $4 \mathrm{H} 8$}

AMS subject classification (1970), Primary 54.35, 54.60, Secondary 54.85.

Key words and phrases, $d$-contraction, Lipschitz constant, uniform contractification, topologically uniformly contractifiable. 